\title{
Optical Positions of Extragalactic Radio Sources Using the $\mathrm{UCAC1}^{1}{ }^{2}$
}

\author{
M. Assafin \\ Depto. de Astronomia - Observatório do Valongo, Universidade Federal \\ do Rio de Janeiro, Rua Ladeira Pedro Antonio, 43 CEP 20080-090 Rio \\ de Janeiro, Brazil, massaf@sun1.ov.ufrj.br \\ N. Zacharias \\ US Naval Observatory, 3450 Massachusetts Avenue, NW, Washington \\ DC 20392
}
A.H. Andrei and R. Vieira Martins
Observatório Nacional - Rua General José Cristino, 77 CEP 20921-400 Rio de Janeiro - RJ, Brazil

\section{Introduction}

Extragalactic radio source positions referred to the first USNO CCD Astrograph Catalog (UCAC1) are presented. They were derived from CCD observations taken with the 1.6-meter Cassegrain telescope at the Laboratório Nacional de Astrofísica, Brazil (LNA). The observations started in 1997 and were carried out in a joint program between USNO, Observatório do Valongo/UFRJ, Brazil, and Observatório Nacional/CNPq, Brazil. They were planned to provide, together with CTIO observations, fiducial points to link the UCAC to the ICRF. The results presented here refer to 24 radio source targets distributed mainly between $-30^{\circ}$ and $-70^{\circ}$ declinations. Reduction techniques follow standard procedures. The results are compared with VLBI and with similar precise optical positions, in order to contribute to the evaluation of the UCAC astrometry.

\section{Observations and reductions}

All CCD positions are referred to a pre-release version of the UCAC1, providing reference stars to $\mathrm{R} \approx 16^{\mathrm{m}}$ with 20 to 70 mas accuracy. The UCAC project and its first catalog are described in Zacharias et al. (2000). CCD observations were taken with the $1.60 \mathrm{~m}$ Cassegrain telescope at LNA, Brazil (f/10; plate scale $=13^{\prime \prime} / \mathrm{mm} ; \lambda=+03^{h} 02^{m} 19.84 ; \phi=-22^{\circ} 23^{\prime} \quad 54^{\prime \prime} .74 ;$ altitude $=1864 \mathrm{~m}$ ). CCD fields were $5^{\prime} \times 5^{\prime}(1050 \times 1050$ pixels of $22.5 \mu \mathrm{m}$ size $)$, with a scale of 0 0!3 per pixel. Three deep exposures per source were obtained $\left(180^{s} \leq \Delta t \leq\right.$ $360^{s}$ ), using a standard $\mathrm{R}$ filter from the UBVR Johnston system. The typical LNA sky brightness is $\mathrm{U}=21.55, \mathrm{~B}=21.99, \mathrm{~V}=21.05, \mathrm{R}=20.39$ and $\mathrm{I}=19.29$. The

\footnotetext{
${ }^{1}$ Based in part on observations made at Laboratório Nacional de Astrofísica/CNPq/MCT

${ }^{2}$ Based in part on observations made at Cerro Tololo Inter-American Observatory (CTIO)
} 


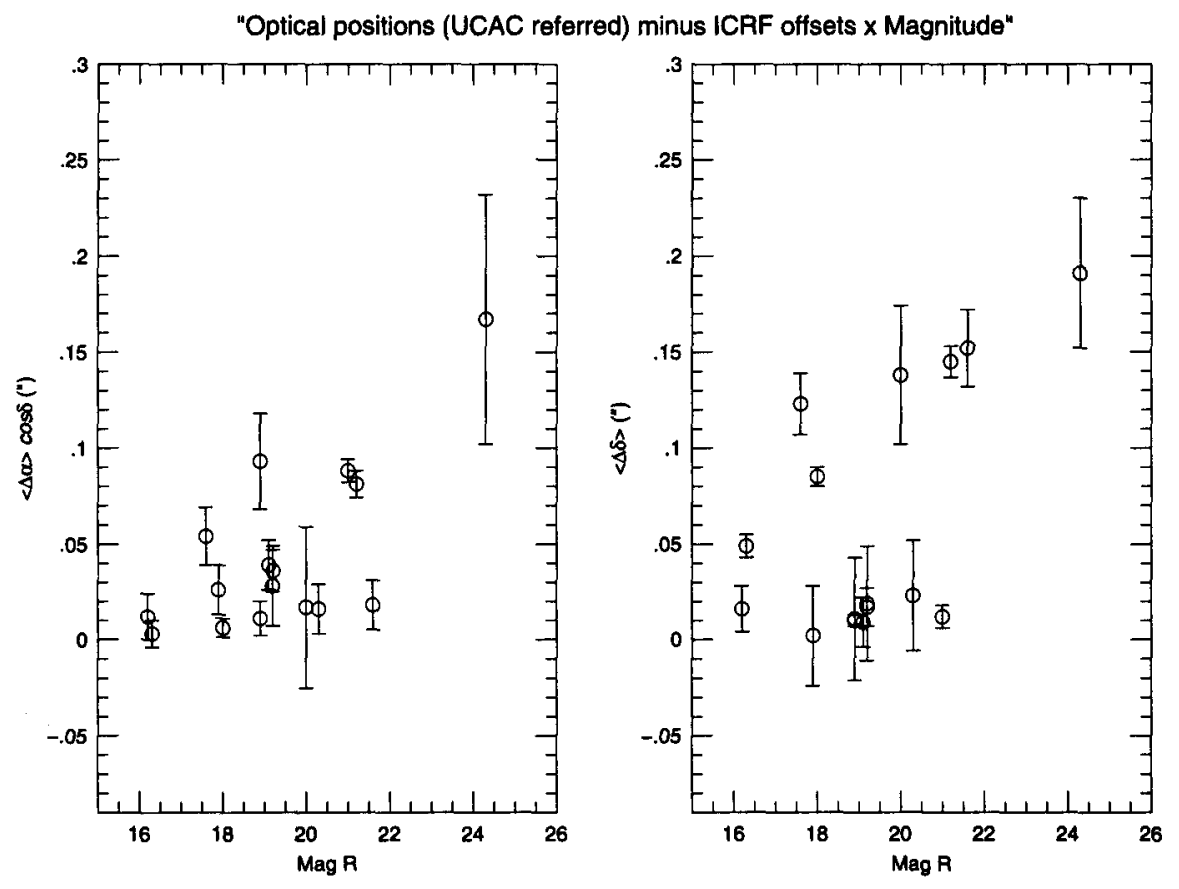

Figure 1. "Optical-radio" position differences versus magnitude.

seeing is typically $1 " 5$ (visual). The raw frames were corrected for bias and flat field. A two dimensional, elliptical Gaussian model ( 7 constants) was adopted to adjust the $(x, y)$ photocenters. The average standard error for an image position is 12 mas per coordinate. Saturation limit is at about $\mathrm{R}=11$, but almost all of the UCAC reference stars on the CCD fields had $R \geq 13$. In order to increase the internal precision, a global reduction technique has been used (see Assafin et al., 1997). The $3 \mathrm{CCD}$ fields are merged into a single, more robust $(\mathrm{x}, \mathrm{y})$ set of measurements, through the use of common tertiary $15 \leq R \leq 19$ stars using a linear adjustment model. The typical mean errors of the CCD frame combination adjustments were 15 mas. The $(\alpha, \delta)$ source positions are then obtained by using the UCAC1 reference stars and a linear model to relate the standard $(\xi, \eta)$ and combined measured $(\mathrm{x}, \mathrm{y})$ coordinates. This procedure gives better results than simply reducing each field separately, and then averaging the individual $(\alpha, \delta)$ coordinates.

\section{Results}

Table 1 shows the results for 24 ICRF target sources. It includes individual source positional errors (standard errors $E_{\alpha}, E_{\delta}$ ), computed from the variancecovariance matrix and the respective $(\mathrm{x}, \mathrm{y})$ center errors added in quadrature, the mean errors $\left(S_{\alpha}, S_{\delta}\right)$ of the $(\alpha, \delta)$ reductions, the $\mathrm{R}$ source magnitudes (estimated from the CCD images), the number of UCAC1 reference stars used in the reductions, and the optical minus ICRF radio position differences. Statistics are summarized at the bottom of the Table. No evident dependence of the 


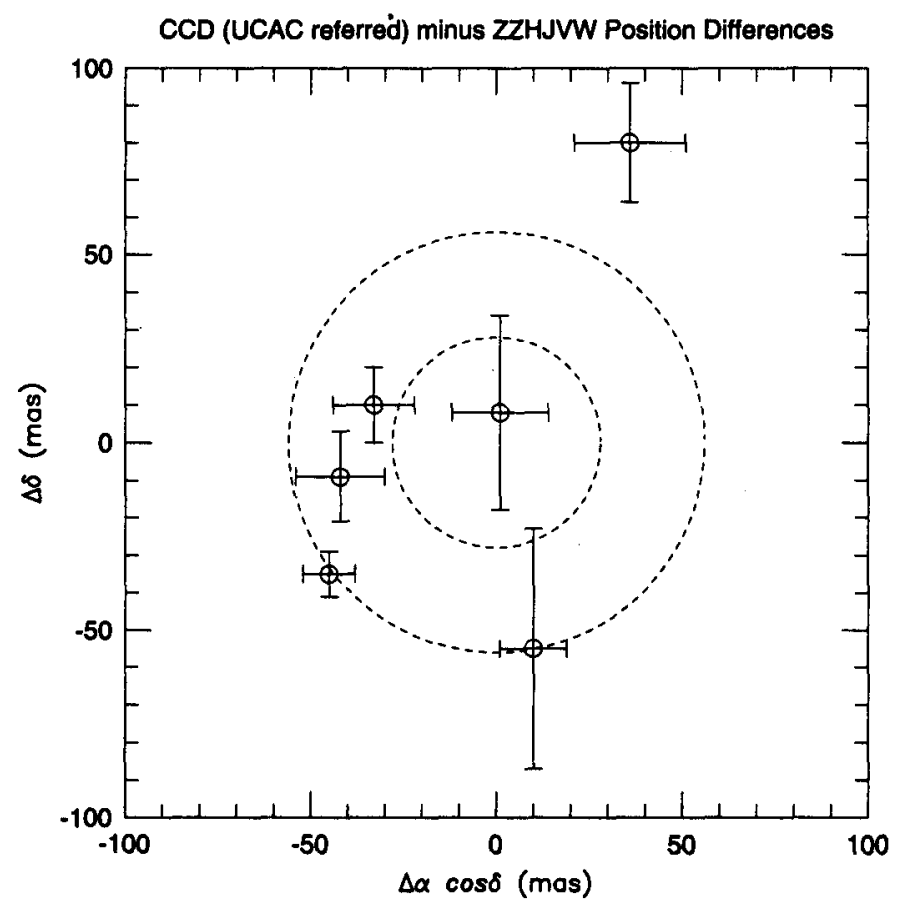

Figure 2. Comparison of optical positions in the sense "this paper minus Zacharias et al. (1999)." Error bars indicate the formal $1 \sigma$ errors from this paper while the dotted circles indicate the $1 \sigma$ and $2 \sigma$ formal position errors from Zacharias et al. (1999). 
"optical-radio" position differences was found as a function of right ascension and declination, or reference star number. Fig. 1 illustrates the "optical-radio" position differences (modulus) against $\mathrm{R}$ magnitudes.

The average "optical-radio" offsets $(\Delta \alpha \cos \delta, \Delta \delta)$ and r.m.s. errors are $-2 \mathrm{mas}(16 \mathrm{mas})$ and $-7 \mathrm{mas}$ (23mas) respectively, for the 16 sources with |optical-radio| $\leq 0^{\prime \prime} 3$. Random subsets of sources gave similar average and r.m.s. values. CCD source images were not detected for 7 targets, with no optical candidates present within $1^{\prime \prime}$ from the radio emission position. Nevertheless, an optical counterpart for source 1251-713 was detected previously with the 0.9-meter CTIO telescope (Zacharias et al. 1999), with the catalog magnitude of $R=20$. This may suggest significant brightness variability for 1251-713.

We have compared the results with other precise optical positions for 6 common sources given in Zacharias et al. (1999). The comparison is illustrated in Fig. 2. The average position differences $(\Delta \alpha \cos \delta, \Delta \delta)$ and r.m.s. errors are -12 mas $(12 \mathrm{mas})$ and -2 mas $(17$ mas $)$ respectively.

Finally, the histogram in Fig. 3 shows the distribution of the standard errors of the source positions (filled line), as well as the distribution of the formal, catalog position errors of UCAC stars within $10^{\prime}$ from the sources (dotted line). Both distributions are alike, indicating that the UCAC precision holds for astrometry of very faint objects.

\section{Future perspectives}

CCD observations of ICRF radio sources still continue with the $1.60 \mathrm{~m}$ LNA telescope and with the USNO CCD Astrograph at CTIO. A final reduction will utilize the CTIO 0.9-meter and astrograph data to provide fainter, secondary reference stars to be used for the reductions of the narrow-field LNA 1.6-meter CCD frames. 

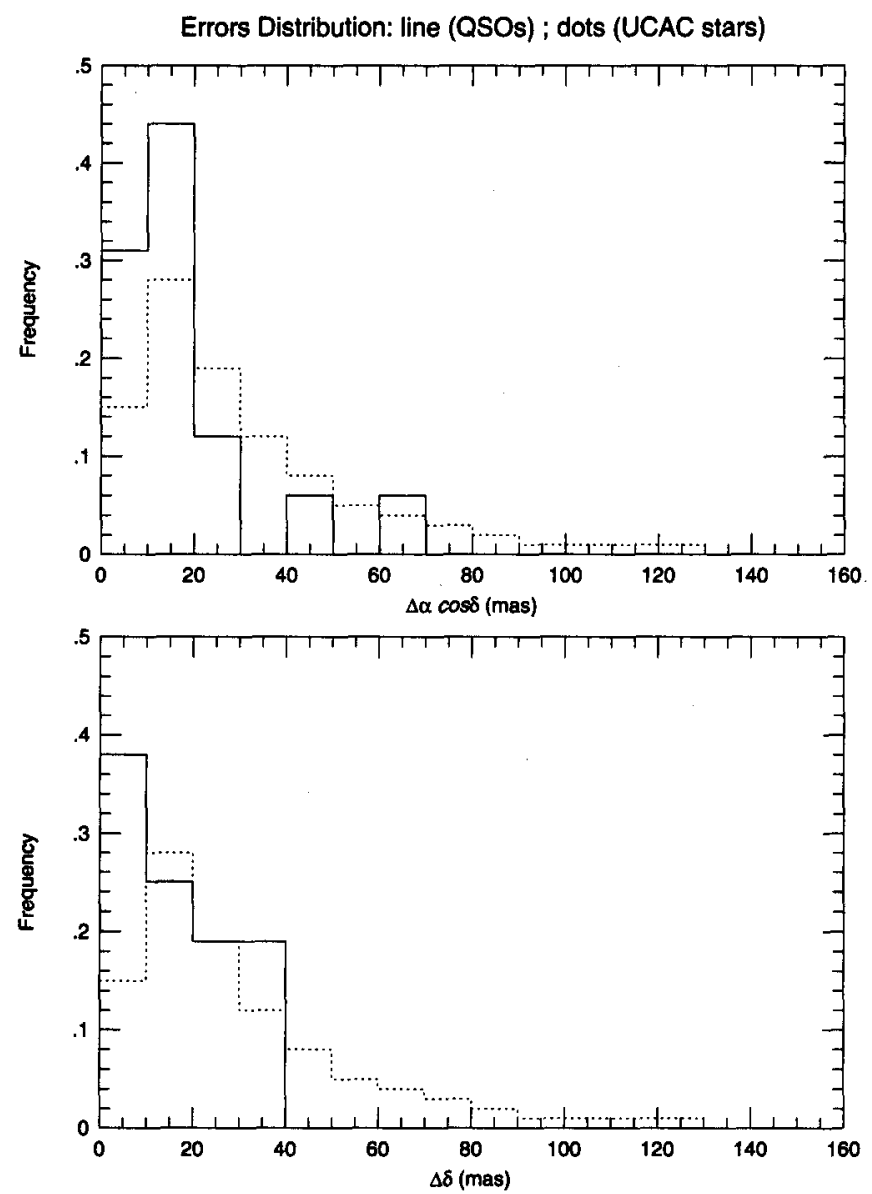

Figure 3. Histogram of the standard error distribution in $(\alpha, \delta)$ of the sources (filled lines), and of UCAC stars (dotted lines) within $10^{\prime}$ from the sources. Results for the RA component (top) and declination (bottom) are shown. 
Table 1. Optical (UCAC referred) minus ICRF Position Results.

\begin{tabular}{|c|c|c|c|c|c|c|c|c|}
\hline ICRF Source & $\begin{array}{c}\text { Optic - } \\
\Delta \alpha \cos \delta \\
\text { (mas) }\end{array}$ & $\begin{array}{c}\text { Radio } \\
\Delta \delta \\
(m a s)\end{array}$ & $\begin{array}{c}\text { Sourc } \\
E_{\alpha} \\
(m a s)\end{array}$ & $\begin{array}{c}\text { Error }^{b} \\
E_{\delta} \\
(m a s)\end{array}$ & $\begin{array}{c}\text { Red } \\
S_{\alpha} \\
(m a s)\end{array}$ & $\begin{array}{c}\operatorname{rror}^{c} \\
S_{\delta} \\
\left(m a_{s}\right)\end{array}$ & $\underset{(R)}{\operatorname{Mag}^{d}}$ & $\begin{array}{c}\text { UCAC }^{e} \\
\text { Stars } \\
\text { No. }\end{array}$ \\
\hline $0517-726^{a}$ & & & & & 39 & 35 & & 10 \\
\hline $0614-349$ & +17 & -138 & 46 & 40 & 35 & 30 & 20.0 & 5 \\
\hline $0646-306$ & +26 & 2 & 14 & 26 & 14 & 30 & 17.9 & 5 \\
\hline $0647-475^{a}$ & & & & & 49 & 50 & & 11 \\
\hline $0700-465$ & +28 & -19 & 24 & 32 & 31 & 44 & 19.2 & 10 \\
\hline $0826-373^{a}$ & & & & & 36 & 23 & & 31 \\
\hline $0936-853$ & -36 & +17 & 16 & 14 & 33 & 31 & 19.2 & 10 \\
\hline $1004-500$ & -81 & +145 & 65 & 62 & 34 & 38 & 21.2 & 29 \\
\hline $1048-313$ & -16 & -23 & 27 & 35 & 26 & 56 & 20.3 & 7 \\
\hline $1101-536$ & -3 & +49 & 09 & 8 & 42 & 40 & 16.3 & 41 \\
\hline $1129-580$ & -6 & +85 & 18 & 19 & 29 & 30 & 18.0 & 42 \\
\hline $1236-684^{a}$ & & & & & 46 & 44 & & 30 \\
\hline $1251-407$ & -18 & -152 & 103 & 188 & 36 & 54 & 21.6 & 9 \\
\hline $1251-713^{a}$ & & & & & 35 & 29 & & 36 \\
\hline $1320-446$ & -39 & -9 & 36 . & 37 & 36 & 35 & 19.1 & 12 \\
\hline $1334-649^{a}$ & & & & & 37 & 45 & & 29 \\
\hline $1424-418$ & +54 & +123 & 19 & 20 & 52 & 53 & 17.6 & 14 \\
\hline $1445-161$ & +11 & +11 & 13 & 33 & 16 & 58 & 18.9 & 5 \\
\hline $1451-375$ & +12 & +16 & 13 & 13 & 41 & 41 & 16.2 & 15 \\
\hline $1600-445$ & +88 & -12 & 42 & 32 & 30 & 32 & 21.0 & 29 \\
\hline $1647-296$ & +193 & +315 & 104 & 220 & 40 & 35 & 20.4 & 23 \\
\hline $1729-373^{a}$ & & & & & 29 & 22 & & 27 \\
\hline $2106-413$ & +93 & -10 & 27 & 10 & 45 & 6 & 18.9 & 4 \\
\hline $2325-150$ & -167 & -191 & 175 & 139 & 55 & 33 & 24.3 & 4 \\
\hline Average & -2 & -7 & 40 & 44 & 36 & 37 & & 18 \\
\hline and r.m.s. & 16 & 23 & 11 & 12 & 2 & 2 & & \\
\hline
\end{tabular}

${ }^{a}$ Source image not detected within 0.77 from radio emission center. Source $1251-713$ has been previously detected in R by Zacharias et al. (1999). See comments in the text.

${ }^{b} E_{\alpha}, E_{\delta}$ : individual source positional errors, computed from the variance-covariance matrix and the respective $(x, y)$ center errors added in quadrature.

${ }^{c} S_{\alpha}, S_{\delta}:$ CCD frame reduction error in $(\alpha, \delta)$ adjustment.

${ }^{d} \mathrm{R}$ source magnitudes (Johnston UBVR system) estimated from our CCD frames.

enumber of UCAC1 reference stars used in the $(\alpha, \delta)$ reductions of the $5^{\prime} \times 5^{\prime}$ CCD fields. 


\section{References}

Assafin, M., Vieira Martins, R., Andrei, A.H., 1997, Astron. J., 113, 1451.

Zacharias, N., Zacharias, M.I., Hall, D.M., Johnston, K.J., de Vegt, C., Winter, L., 1999, Astron. J., 118, 2511.

Zacharias, N. et al., 2000, Astron. J. (submitted). 